\title{
The Design of Greenhouse Environment Control System Based on LabVIEW and ZigBee
}

\author{
Lei Wang \\ Information Engineering of Tangshan College, Tang Shan, China \\ e-mail: wanglei122_2000@126.com
}

\begin{abstract}
The Internet of things technology widespread application in the management of agricultural greenhouse. In this paper, research finding on the greenhouse environment management in tangshan laoting county modern NongYeYuan, which is based on LabVIEW and ZigBee. System is divided into upper desktop module and desktop module, which is controld by STC. The sensors function is to acquisition environment parameters of greenhouse, transmit it by ZigBee wireless module. Alarm information is transferred to user by GSM, and initial value can also report to the police to set the module and the lower limit of the ceiling. System is divided into auto and manual mode, which is realized on the mode switch. Through the field test and operation, the system is proved to be reliable stable, and it has practicability and generalization.
\end{abstract}

Keywords-LabVIEW; ZigBee; GSM; STC; Monitoring system.

\section{INTRODUCTION}

With the continuous development of the Internet of things technology, agricultural science and technology modernization level gradually improve. Intelligent ecosystem environment parameter control is extremely important in crops growth[1]. Tangshan greenhouse planting area is the first in the province. The monitoring measures are mostly used by manual in the greenhouse, which is difficult to guarantee the real time capability and effectiveness of the data. Also it difficult to wire transmission because of the limitation of the physical condition. Thus it is necessary to use proper wireless transmission ${ }^{[2]}$. According to the present situation, the intelligent control system for greenhouse environment monitoring is designed. This system makes use of the ZigBee which realizes the data wireless transmission between the two position machines. This method can effectively reduce the geographical position, physical lines and complex environmental factors, improve the transmission efficiency. System adopts LabVIEW design human-computer interaction interface, easy to management and stable operation.

\section{THE DIAGRAM OF THE SYSTEM OVERALL DESIGN}

Crops in the growth process requires a lot of environmental factors, the appropriate temperature, humidity, light intensity and CO2 concentration is the key to achieve healthy growth of crops. Therefore, combined with the crop growth rule, the greenhouse environment monitoring and control, to realize the detection and adjustment of greenhouse environment.

The design is divided into data acquisition module, data transmission module and upper desktop interface control module three parts. In this system, STC as the core of the control module, to achieve and transmission of information of desktop. Data acquisition system includes a data acquisition and processing, display and data transmission and other functions. Data acquisition, including temperature and humidity of air and soil, light intensity and $\mathrm{CO} 2$ concentration, will send the collected data to ZigBee endnode of transmission system. ZigBee end-node will Transfer the data received to ZigBee network coordinator, ZigBee network coordinator Transfers the data to PC,PC upper desktop will display the data collected in real time, and comparing with setting threshold, When it exceeds the set upper and lower limits, there will be a red indicator showing directly on the upper desktop, upper desktop interface adjustment mechanism includes measures to automatic adjustment and manual adjustment, the automatic mode can complete alarm messages and pass corresponding improvement in the greenhouse environment instruction to the desktop to adjust, while the lower computer buzzer sounding, indicating that the adjustment mechanism is in progress, an administrator at the computer, can be set to manual adjustment mode, according to the data displayed, click on the appropriate button to adjust the measures, then perform the appropriate adjustment institutions. When administrators away from the computer, open the automatic adjustment mode, the upper desktop automatically sends alarm messages to the designated administrators, and perform the appropriate adjustment institutions. Administrators can connect to the Internet, log on to the administration interface for real-time monitoring, modifying the upper and lower limits, mediation and other operations. The overall design diagram contains three scenes, which are among the scene, the computer room and the mobile terminals, the site is transferred to the computer room wirelessly through ZigBee, the computer room and the mobile terminals communicate via GSM network. The design of the overall block diagram shown in Figure 1.

This paper introduces the composition of the system from two aspects of hardware design and the upper desktop software control. The user can monitor the greenhouse environment parameters conveniently through this system. 


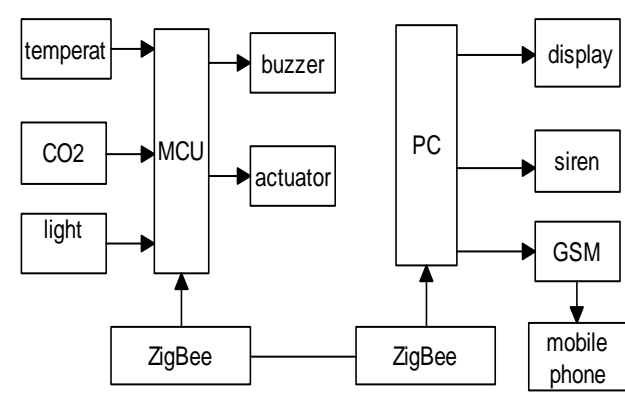

Figure 1. The diagram of the system overall design.

\section{The Design OF THE DESKTOP}

The system uses on STC MCU as the control chip, the desktop main program including temperature and humidity data acquisition module, light intensity acquisition module, a $\mathrm{CO}_{2}$ collection module, LCD display module, reset circuit, a buzzer circuit, analog-to-digital conversion circuit modules, together to complete the function of the data acquisition, transmission and regulation. The main diagram is shown in Figure 2.

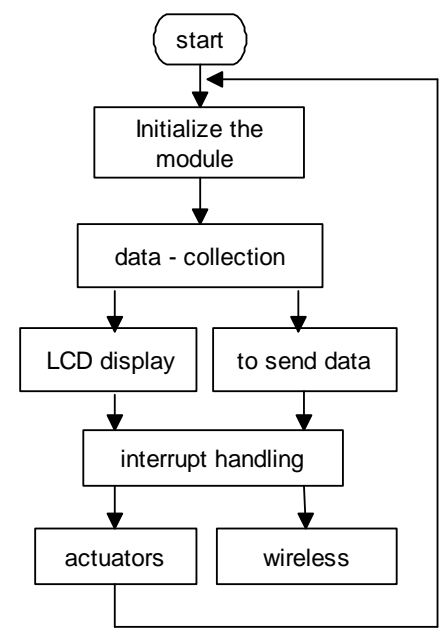

Figure 2. The diagram of desktop.

STC MCU P2 port contains P2.0 to P2.7, pin 21 to 28, external LCD display, where pins 24, 25, 26 respectively control the monitor data command, read and write, enable function. P3.0 to P3.7, external serial port, buzzer, temperature and humidity, light intensity and $\mathrm{CO}_{2}$ concentration and other circuits, is to realize the function of the most part.P0.0 to P0.7 connect resistor network connect LCD to display outside, to control the contents of the display part.P1.0 to P1.7, pin 2 to 8 connect seven LED external, representing different regulation mechanism, the pin 2 controls the blower, the pin 3 controls the heat, pin 4 controls damping, pin 5 controls irrigation, pin 6 controls top window, pin 7 controls shading, pin 8 controls the fill light relay switch. The System chooses DHT11 as the acquisition module of air temperature and humidity degree, and the output end connected to the MCU P3.5, soil environment temperature and humidity data connects with the MCU P3.7 through DHT11 data interface, then transfers digital signal into the MCU for processing.

\section{NETWORK FORM AND SERIAL CONTROL OF ZIGBEE}

ZigBee is synonymous with IEEE 802.15.4 protocol. ZigBee protocol stack refers to the network layer protocol in the sum of its image reflecting a network file transfer process: by the upper level protocol to the underlying, and by the underlying protocol to the upper ${ }^{[3]}$. This design uses the CC2530F256 with 256 KB of flash memory. CC2530F256 combines ZigBee protocol stack, providing a good ZigBee development platform. The design of this system uses ZigBee technology as a wireless transmission parts, achieving the point-to-point communication with two CC2530 modules, a coordinator, connected to the PC, another terminal equipment, connected with the machine. ZigBee coordinator is a device to initiate and configure the network. Coordinator can keep indirect addressing with binding forms, support association. When using it, open the coordinator first, and then open the terminal equipment.

This system adopts the question and answer form to achieve the communication between the upper and lower computer. The upper computer sends command characters, after receive the character, the lower computer transfer 6 number to the upper computer, and control correspondingly according to whether the character is a control character. The benefits of this design, on the one hand, avoid the conflicts of transmission data from top to bottom, making the system more stable; On the other hand, it saves the resources of the lower computer and the transmission system.

Serial port sends six data values, two monitoring points' temperature and humidity, light intensity and $\mathrm{CO}_{2}$ density. Initialize the serial port and set the baud rate and other parameters before sending. Select work style of SCON, set baud rate double "SMOD" (default $=0$ ) for the PCON, use interrupt way, open the ES serial port interrupt bit and the total interrupt EA bit. Figure 3 shows a serial port and control program flow.

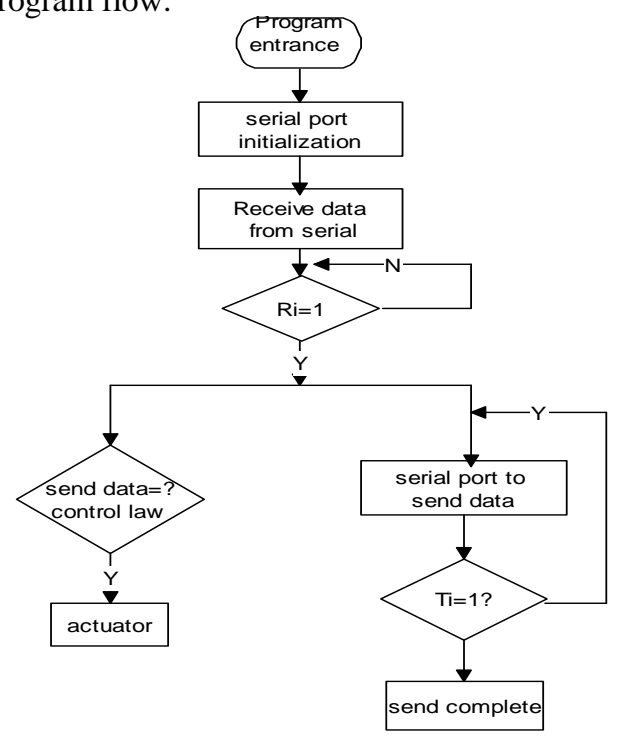

Figure 3. Serial port and control program flow. 


\section{THE USER INTERFACE DESIGNED BY LABVIEW}

LabVIEW is a software development environment with relatively complete function. However it is not just a software development environment for it is designed to alternate the regular Basic or C language. As a language for developing applications, LabVIEW has all the characteristics of programming languages except programming style, so it is called the $\mathrm{G}(\mathrm{Graph})$ language. When programming with the $\mathrm{G}$ language, users usually use flow chart or block diagram instead of writing program code ${ }^{[4]}$. The interface of this upper computer adopt the LabVIEW program with the following functions: username and password login; display of the air and soil temperature and humidity, light intensity, carbon dioxide density; historical data collection, waveform display; alarm of the comparison between the instantaneous value of the corresponding point with the upper and lower limit value; adjusting the control of the lower computer; control of GSM module.

The main interface includes a serial port selection section, an administrator phone number and text message receiving section, real-time data collection and upper and lower display, automatic and manual control section, historical data recording section, real-time waveform data display section. The main interface of LabVIEW is shown in Figure 4,5and6.The design of login system is based on LabVIEW software platform, and its main function is to ensure security of users and the test data. The screen before the login is shown in Figure 7.

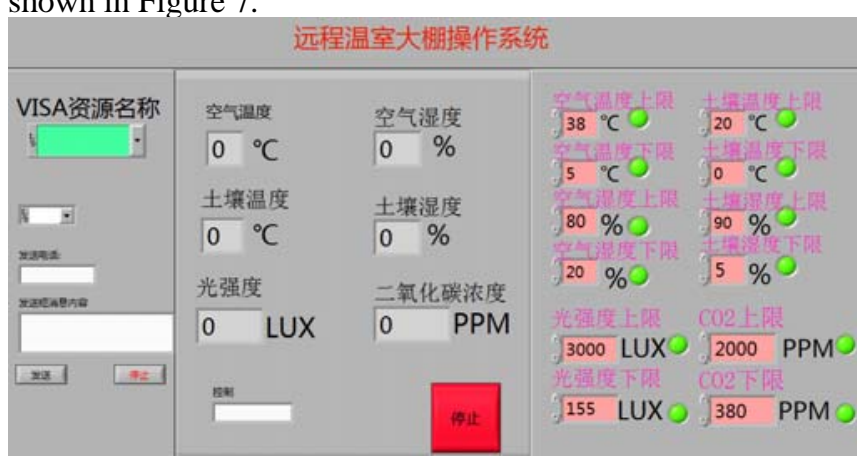

Figure 4. LabVIEW front panel main industry.

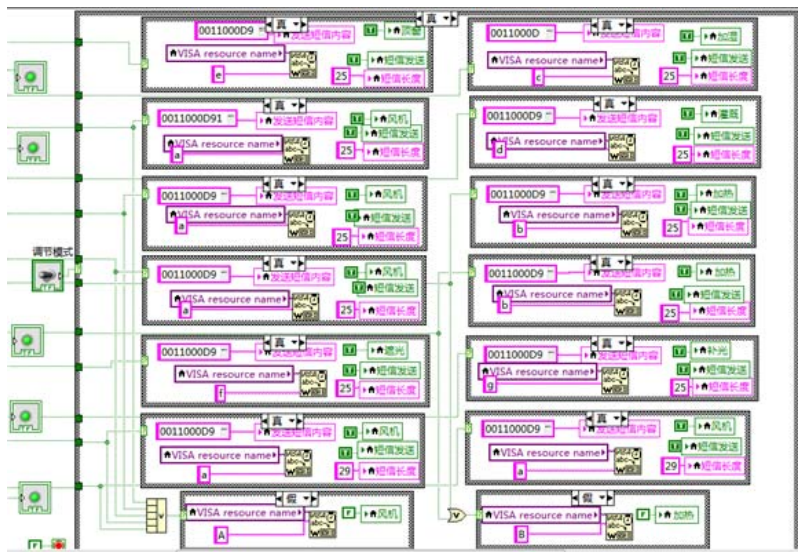

Figure 5. Automatic adjustment mode.

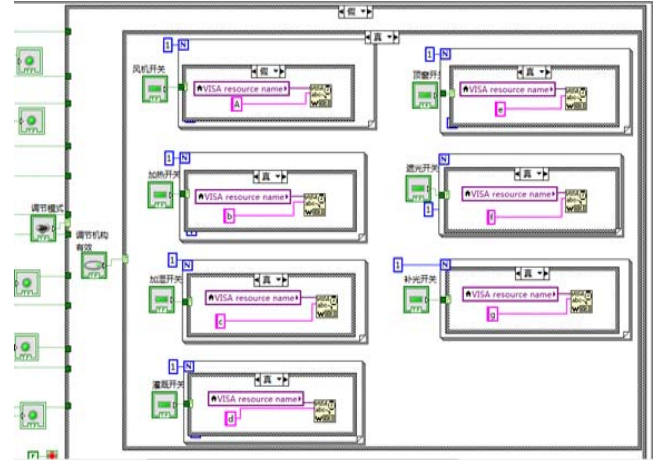

Figure 6. Manual regulation mode.

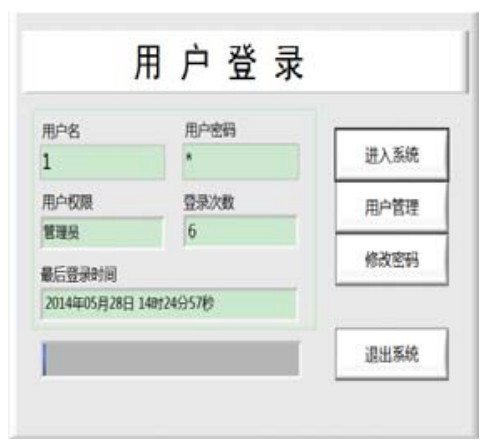

Figure 7. Before login interface VI interface.

This system can be realized within the greenhouses on air temperature and soil temperature, light intensity and $\mathrm{CO}_{2}$ concentration and other numerical continuous monitoring. System data wireless transmission is realized using the ZigBee, conform to the requirements of the use of modern greenhouse, saves the manpower, the material resources of the greenhouse environment monitoring and control more scientific. Upper machine has a good interface, realize the serial communication, display, alarm, and sending short message function. System has automatic and manual control mode, it can real-time remote monitoring of the greenhouse environment. Automatic and manual mode with the combination of good system added flexibility and extensibility. System through field test, low power consumption, stable running well, user operation is simple, has a wide applicability.

\section{REFERENCES}

[1] Zhang xiaolong. The design and implementation of agricultural greenhouse control system based on ZigBee [D]. Beijing university of posts and telecommunications, 2009:26-29

[2] Li shihui. The research on the intelligent greenhouse monitoring system based on wireless sensor network [J]. Journal of Jiangxi University of science and technology. 2013(34):71-73.

[3] Cai bin. Research and design of agricultural environment monitoring system based on ZigBee wireless sensor network [J]. Acta agriculturae Jiangxi. 2010(11):153-156.

[4] Xu dong.Design of wireless sensor network node for environment monitoring based on CC2530 [J]. Journal of computer applications. 2013(33):17-20. 
[5] Chen xiaoyan. Design on water-saving irrigation system of greenhouse based on ZigBee networks [J]. Transducer and microsystem technologies. 2013(32):82-85

[6] Wang shouhua. Field testing application in modern agriculture based on wireless sensor and GSM [J].Journal of anhuig agri.sci.2011, 39(32):20254-20255.
[7] Sun liting. The design of greenhouse monitoring system based on WSN [D]. Dalian university of technology, 2013:12-15

[8] Wang dong. Research and realization control system of greenhouse environment based on MUTI sensor fusion [D]. Northwest A\&F University, 2012:32-34. 